14.02

\title{
Оптимизация параметров импульсных РЧ-последовательностей для МРТ-исследований в присутствии магнитных наночастиц
}

\author{
( Ю.В. Богачев, ${ }^{1}$ А.В. Никитина, ${ }^{1}$ В.В. Фролов, ${ }^{2}$ Я.Ю. Марченко, ${ }^{1}$ Б.П. Николаев ${ }^{1}$ \\ ${ }^{1}$ Санкт-Петербургский государственный электротехнический университет (ЛЭТИ), \\ 197376 Санкт-Петербург, Россия \\ ${ }^{2}$ Санкт-Петербургский государственный университет, \\ 198504 Санкт-Петербург, Россия \\ e-mail: nastya_nikitina1996@mail.ru, yu.bogachev@mail.ru \\ Поступило в Редакцию 19 декабря 2019 г. \\ В окончательной редакции 19 декабря 2019 г. \\ Принято к публикации 17 февраля 2020 г.
}

\begin{abstract}
Рассмотрены вопросы влияния параметров различных импульсных радиочастотных (РЧ) последовательностей на контраст магнитно-резонансных (MP) изображений. Разработана программа для моделирования магнитно-резонансных томограмм и определения оптимальных значений параметров импульсных РЧпоследовательностей для достижения наилучшего контраста МР-изображений. Данная программа апробирована для МРТ-исследований с использованием негативных контрастирующих агентов на основе магнитных наночастиц оксида железа и позитивных контрастирующих агентов на основе гадолиния. Проанализировано их влияние на интенсивность МР-сигнала в зависимости от концентрации. Разработаны рекомендации по выбору оптимальных значений параметров РЧ-последовательностей для МРТ-диагностики с использованием контрастирующих агентов.
\end{abstract}

Ключевые слова: МРТ, импульсные радиочастотные последовательности, магнитные наночастицы, тераностика.

DOI: 10.21883/JTF.2020.09.49678.419-19

\section{Введение}

Магнитно-резонансная томография (МРТ) - признанный метод современной неинвазивной медицинской диагностики [1 4$]$. Благодаря данному методу появилось новое интенсивно развивающееся направление - магнитно-резонансная (MP) тераностика, в котором современные методы диагностики сочетаются с терапией $[5,6]$. Для этого в МРТ используются новые мультимодальные контрастирующие агенты на основе гадолиния или магнитных наночастиц оксида железа. Эти тераностические наночастицы должны не только обладать высокой эффективностью ЯМР-релаксации, но и быть стабильными, биосовместимыми, нетоксичными, обладать способностью к функционализации для взаимодействия с определенными биологическими объектами.

Для повышения информативности и качества магнитно-резонансных томограмм в присутствии мультимодальных контрастирующих агентов необходимо улучшение контрастности МР-изображений. Управлять контрастом можно с помощью изменения параметров импульсных радиочастотных (РЧ) последовательностей МРТ-сканера [1,7].

Поэтому моделирование магнитно-резонансных томограмм, анализ и оптимизация параметров применяемых импульсных РЧ-последовательностей актуально для проведения МРТ-исследований в присутствии новых тераностических наночастиц.

\section{1. Методы исследований}

Для моделирования магнитно-резонансных томограмм в присутствии тераностических наночастиц была исследована ЯМР-релаксация протонов водных растворов магнитных наночастиц на основе оксида железа. В качестве объектов исследования были синтезированы магнитные наночастицы оксида железа в оболочке декстрана. Наночастицы оксида железа получали совместным осаждением растворов солей двухвалентного и трехвалентного железа с избытком щелочи в присутствии $\mathrm{CsCl}$ при $80^{\circ} \mathrm{C}$ в атмосфере $\mathrm{N}_{2}$ по схеме реакции:

$$
\begin{aligned}
& \mathrm{FeSO}_{4} \cdot 7 \mathrm{H}_{2} \mathrm{O}+2\left(\mathrm{FeCl}_{3} \cdot 6 \mathrm{H}_{2} \mathrm{O}\right)+8 \mathrm{NH}_{4} \mathrm{OH} \\
& =\mathrm{Fe}_{3} \mathrm{O}_{4} \downarrow+6 \mathrm{NH}_{4} \mathrm{Cl}+\left(\mathrm{NH}_{4}\right)_{2} \mathrm{SO}_{4}+23 \mathrm{H}_{2} \mathrm{O} .
\end{aligned}
$$

Затем был введен стабилизатор для предотвращения агрегации магнитных наночастиц и для повышения стабильности дисперсии. Для стабилизации нанодисперсного оксида железа использовали $30 \%$ раствор декстрана $(9-11 \mathrm{kDa})$. Стабилизация достигалась ультразвуковым воздействием.

Измерение времен ЯМР-релаксации и запись спектров ЯМР проводились на ЯМР-спектрометре высокого разрешения CXP-300 (Bruker, Германия) с магнитным полем 7.1 Т. Измерения проводились при температуре $23 \pm 2{ }^{\circ} \mathrm{C}$.

Длительность $180^{\circ}$-импульса составляла $26 \mu \mathrm{s}$, длительность $90^{\circ}$-ого импульса - 13 mus. Измерение вре- 


\begin{tabular}{c|c|c|c}
\hline$C, \mathrm{mMol} / 1$ & $T_{1}, \mathrm{~ms}$ & $T_{2}, \mathrm{~ms}$ & $T_{2}^{*}, \mathrm{~ms}$ \\
\hline 0.1 & 1956 & 18.62 & 8.61 \\
\hline 0.5 & 868 & 4.10 & 2.63 \\
\hline 1 & 508 & 3.01 & 1.52 \\
\hline 2 & 287 & 1.48 & 0.81 \\
\hline 4 & 149 & 0.75 & 0.43 \\
\hline 8 & 78 & 0.38 & 0.24 \\
\hline 10 & 63 & 0.34 & 0.19
\end{tabular}

мени спин-решеточной релаксации $T_{1}$ осуществлялось при помощи импульсной последовательности „инверсия-восстановление“ $\left(180^{\circ}-\tau-90^{\circ}\right)$. Значение времени $\tau$ подбиралось эмпирическим путем для каждой точки и находилось в интервале значений от $100 \mu \mathrm{s}$ до $15 \mathrm{~s}$. Измерение времени спин-спиновой релаксации $T_{2}$ осуществлялось при помощи импульсной последовательности „Кара-Парселла-Мейбума-Гилла“

$$
\left(90^{\circ}-\tau-180_{x}^{\circ}-2 \tau-180_{-x}^{\circ}-2 \tau-180_{x}^{\circ}-2 \tau-180_{-x}^{\circ}-\tau\right) .
$$

Время поперечной релаксации $T_{2}^{*}$ рассчитывали по ширине линии протонного спектра ЯМР исследуемого образца в соответствии с выражением

$$
T_{2}^{*}=\frac{1}{\pi \Delta v_{\frac{1}{2}}^{\text {meas }}},
$$

где $\Delta v_{\frac{1}{2}}^{\text {meas }}$ - ширина спектральной линии ЯМР, измеренная

В таблице приведены полученные значения времен ЯМР-релаксации водных растворов в присутствии магнитных наночастиц, которые использовались в дальнейшем моделировании.

ЯМР-релаксационные характеристики водных растворов в присутствии магнитных наночастиц

С целью оптимизации контраста МР-изображений были смоделированы и проанализированы зависимости интенсивности МР-сигнала от концентрации контрастирующих агентов для трех часто используемых в МРТ импульсных РЧ-последовательностей.

Интенсивность регистрируемого в МРТ сигнала пропорциональна поперечной намагниченности $M_{y}^{\prime}$ во вращающейся системе координат. Величина поперечной намагниченности в последовательности „спиновое эхо“ определяется по следующей формуле [7]:

$$
\begin{aligned}
& M_{y^{\prime}}=M_{0} e^{-T_{E} / T_{2}} \sin \alpha \\
& \times \frac{1+\exp \left(-T_{R} / T_{1}\right)-2 \exp \left(-\left(T_{R}-T_{E} / 2\right) / T_{1}\right)}{1+\exp \left(-T_{E} / T_{2}\right) \cos \alpha},
\end{aligned}
$$

где $M_{0}-$ величина намагниченности в начальный момент времени, $T_{R}$ - время повторения между $90^{\circ}$-импульсами, $T_{E}-$ время эхо, $T_{1}-$ время спин-решеточной релаксации, $T_{2}-$ время спин-спиновой релаксации, $\alpha-$ угол отклонения.

Для последовательности „инверсия-восстановление“ величина поперечной намагниченности была определена как [1]:

$$
M_{y^{\prime}}=M_{0}\left(1-2 \exp \left[-T_{I} / T_{1}\right]+\exp \left[-T R / T_{1}\right]\right),
$$

где $M_{0}-$ величина намагниченности в начальный момент времени, $T_{I}-$ время инверсии.

Для последовательности $F L A S H$ величина поперечной намагниченности определялась по формуле [6]:

$$
M_{y^{\prime}}=M_{0} \frac{\left(1-\exp \left(-T R / T_{1}\right)\right) \sin \alpha \exp \left(-T E / T_{2}^{*}\right)}{1-\exp ()-T R / T_{1} \cos \alpha},
$$

где $\alpha-$ угол отклонения, $T_{R}-$ время повторения, $T_{E}-$ время эхо, $T_{1},, T_{2}^{*}-$ времена релаксации.

\section{2. Результаты исследований}

Для проведения сравнительного анализа смоделированных МР-томограмм были определены значения интенсивности МР-сигнала для различных концентраций магнитных наночастиц при фиксированных параметрах для указанных выше трех последовательностей РЧ-импульсов.

В качестве фиксированных были приняты следующие параметры радиочастотных последовательностей:

- последовательность „спиновое эхо“: $T_{E}=10 \mathrm{~ms}$ и $T_{R}=2000 \mathrm{~ms}, \alpha=90^{\circ}$;

- последовательность „инверсия-восстановление“: $T_{I}=1200$ ms и $T_{R}=2000 \mathrm{~ms}$;

- последовательность FLASH: $T_{R}=100 \mathrm{~ms}$, $T_{E}=10 \mathrm{~ms},-\alpha=60^{\circ}$.

Данные параметры не являются оптимальными, однако такие значения были выбраны намеренно для наглядной иллюстрации изменения интенсивности МР-сигнала в зависимости от концентрации магнитных наночастиц.

Были построены зависимости интенсивности МР-сигнала от концентрации магнитных наночастиц (рис. 1) для: импульсной последовательности „спиновое эхо“ $(a)$, импульсной последовательности $F L A S H(b)$, импульсной последовательности „инверсия-восстановление“ $(c)$.

Из зависимостей, приведенных на рис. 1 , можно сделать вывод, что исследуемые магнитные наночастицы значительно уменьшают интенсивность сигнала на $T_{2}$ взвешенных томограммах в области аккумулирования контрастирующих агентов. В этом случае интенсивность сигнала в большей степени зависит от времени спинспиновой релаксации $T_{2}$. Данное снижение интенсивности сигнала характеризует исследуемые магнитные наночастицы как хорошие негативные контрастирующие агенты.

Для практического использования магнитных наночастиц на основе оксида железа в качестве негативных контрастирующих агентов необходимо определить оптимальные параметры импульсных последовательностей 

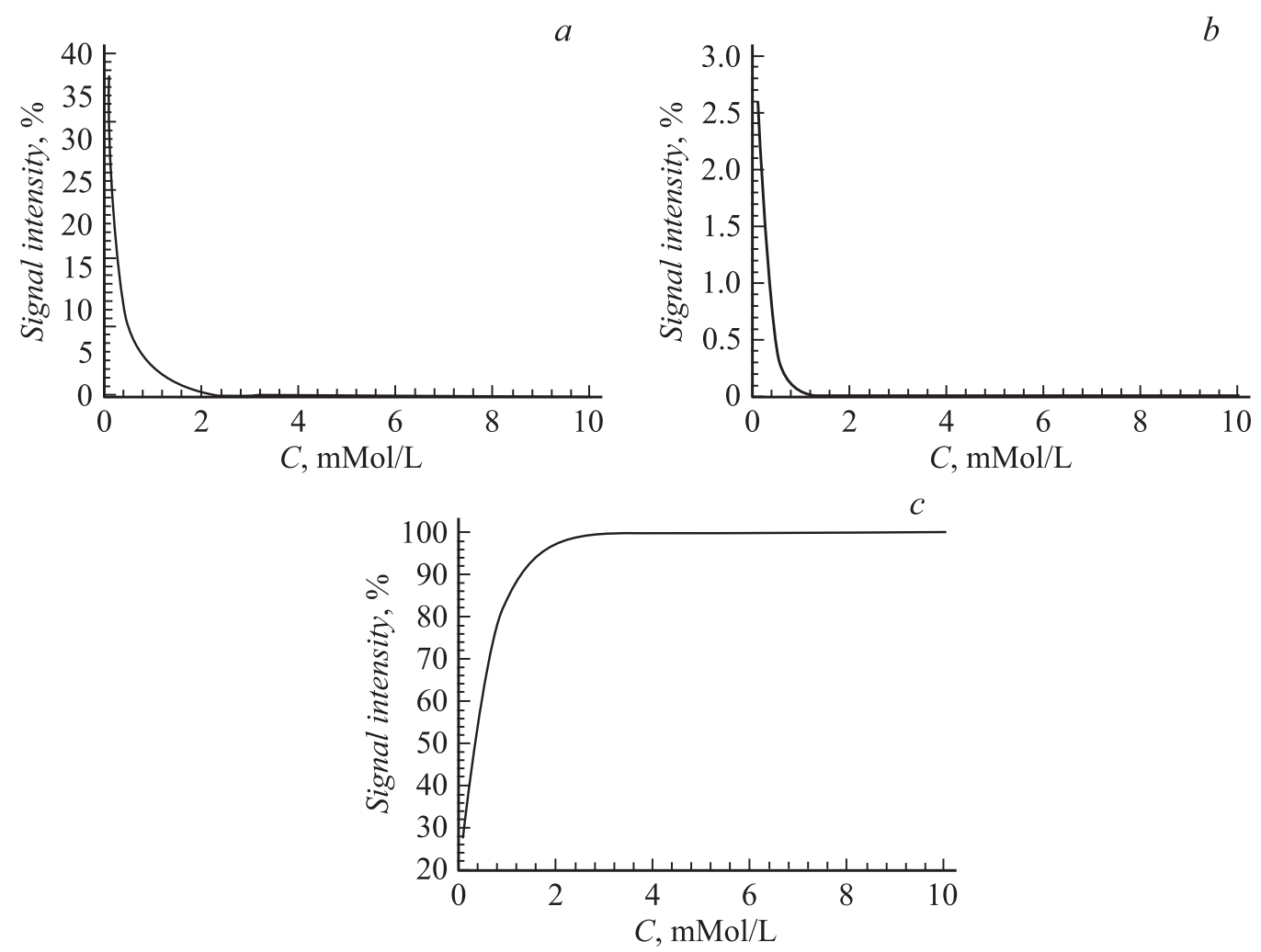

Рис. 1. Зависимости интенсивности МР-сигнала от концентрации магнитных наночастиц.
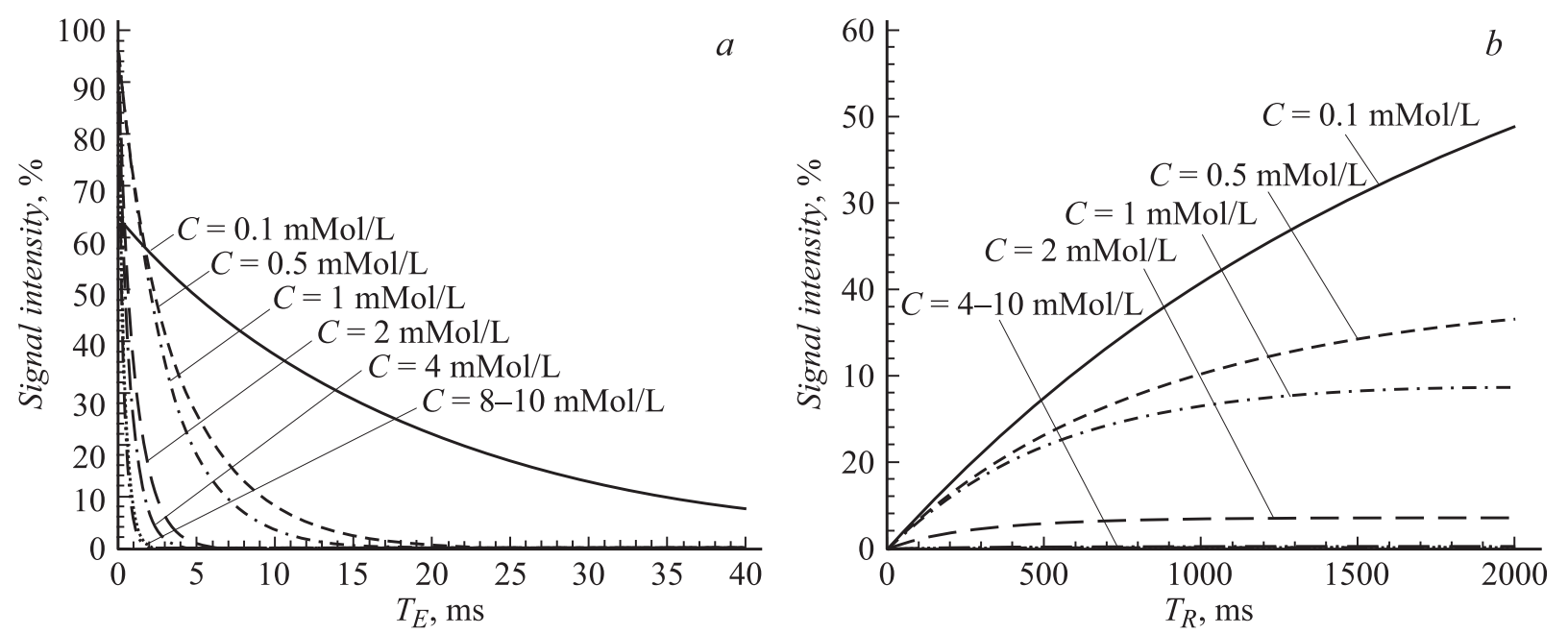

Рис. 2. Зависимости интенсивности МР-сигнала от параметров последовательности „спиновое эхо“.

для различных концентраций данных агентов. Были исследованы зависимости интенсивности МР-сигнала от параметров трех импульсных РЧ-последовательностей и сформулированы рекомендации по выбору оптимальных значений этих параметров для достижения наилучшего контраста МР-изображений в присутствии контрастирующих агентов. На рис. 2 представлены зависимости интенсивности МР-сигнала от параметров: $T_{E}(a)$ и $T_{R}(b)$ последовательности „спиновое эхо“для различных концентраций магнитных наночастиц.
По зависимостям, приведенным на рис. 2, можно сделать вывод, что при малых значениях времени эха исследуемые магнитные наночастицы будут несколько ослаблять сигнал в месте их накопления. При этом чем больше концентрация наночастиц, тем сильнее они влияют на интенсивность сигнала. Из графика на рис. 2, $a$ видно, что для значительного подавления МР-сигнала в области накопления контрастирующего агента, а значит, и для лучшего контрастирования данной области, необходимо выбрать значение времени эха более $40 \mathrm{~ms}$. 

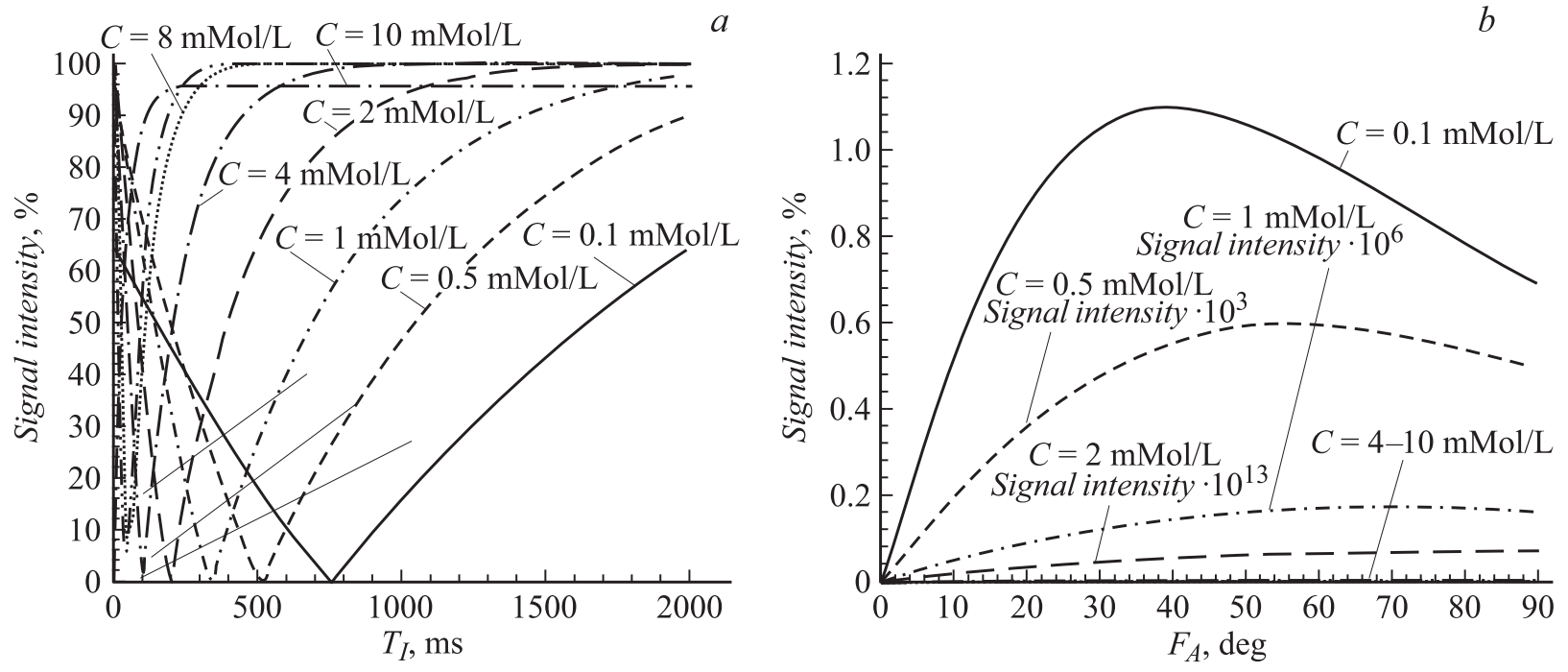

Рис. 3. Зависимости интенсивности МР-сигнала от параметров последовательностей „инверсия-восстановление“ и $F L A S H$.

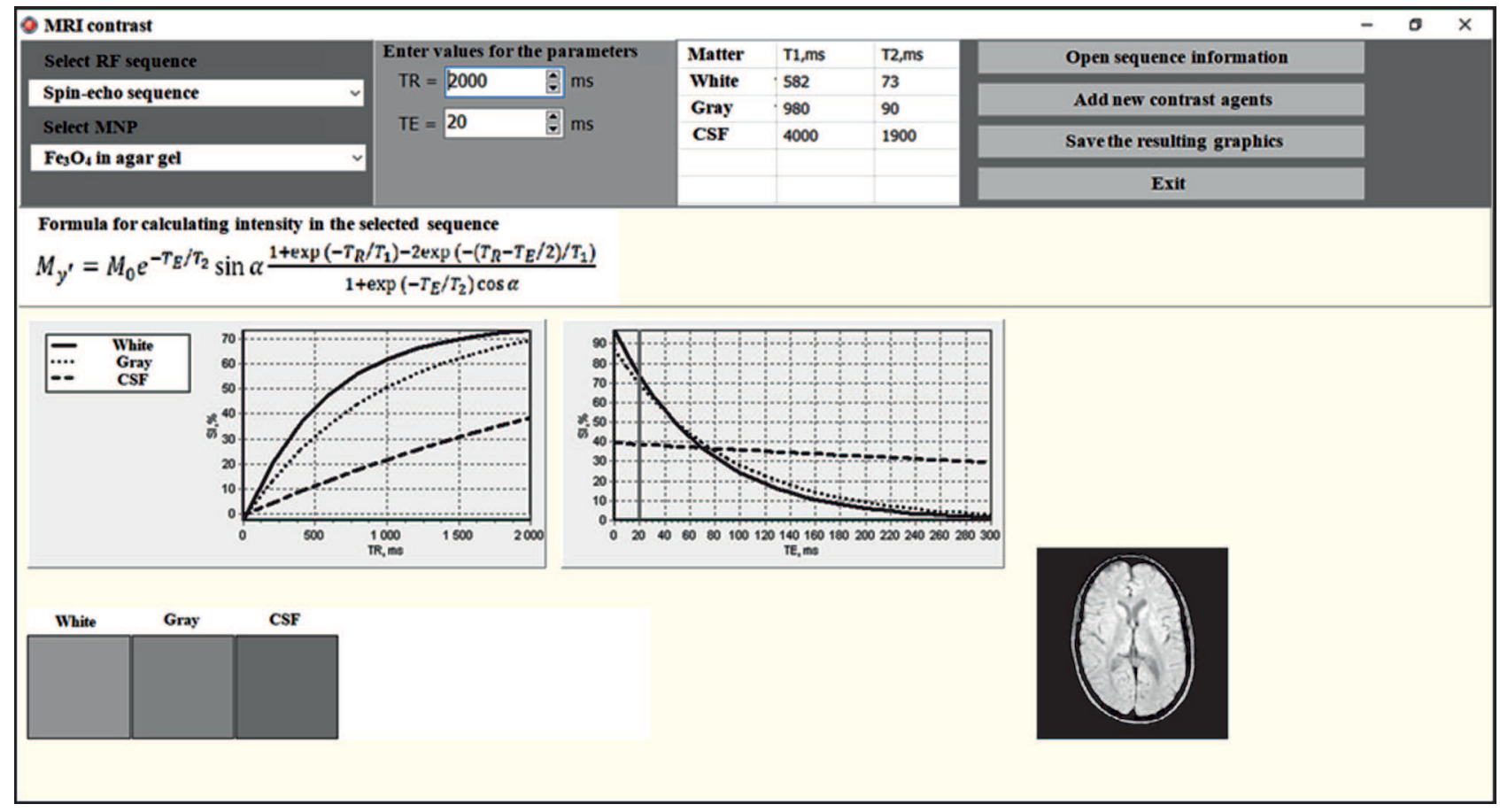

Рис. 4. Рабочее окно разработанной программы.

Из графика на рис. 2, $b$ видно, что для лучшего подавления сигнала необходимо увеличивать концентрацию наночастиц.

На основании данных по ЯМР-релаксации, представленных в таблице, были построены зависимости интенсивности МР-сигнала от параметров: $T_{I}$ - времени инверсии для последовательности „инверсия-восстановление“ (рис. 3,a) и $\alpha(F A)$ - угла отклонения для последовательности FLASH (рис. 3,b) для различных концентраций магнитных наночастиц.
Из зависимостей, приведенных на рис. 3, $a$ видно, что с увеличением времени инверсии интенсивность МР-сигнала возрастает. В последовательности „инверсия-восстановление“, интенсивность сигнала определяется, главным образом, временем спин-решеточной релаксации $T_{1}$, а это значит, что для достижения наилучшего контраста изображения на $T_{1}$-взвешенных томограммах должно быть выбрано $T_{I}>2000 \mathrm{~ms}$.

При рассмотрении зависимости интенсивности МРсигнала от угла поворота $\alpha(F A)$ для последовательности $F L A S H$, можно заключить, что для хорошего подавления 


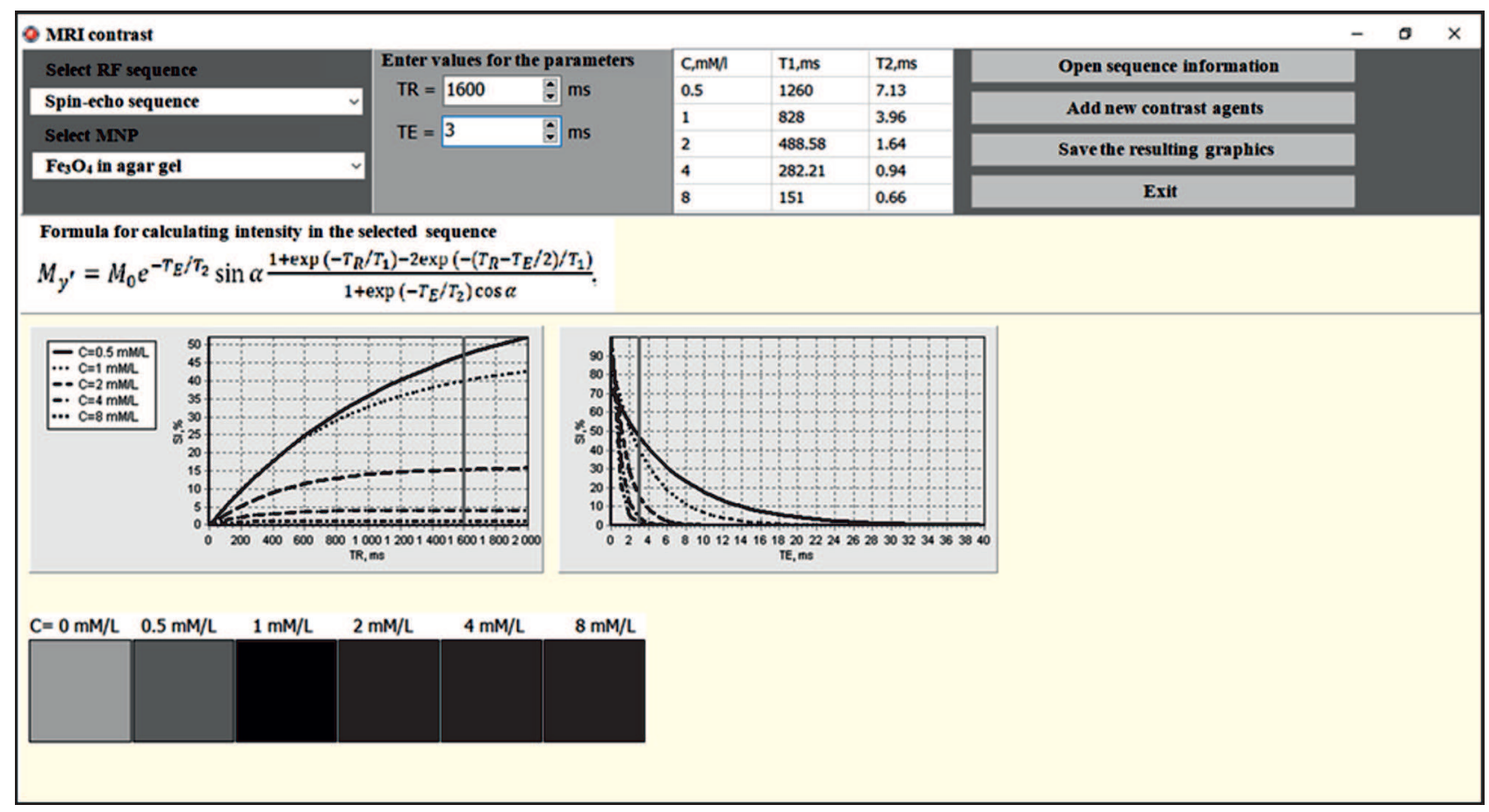

Рис. 5. Рабочее окно разработанной программы для МРТ с использованием магнитных наночастиц.

сигнала на $T_{2}$-взвешенных томограммах необходимо выбрать углы поворота $\alpha<20^{\circ}$ или $\alpha>60^{\circ}$. При высоких концентрациях магнитных наночастиц влияние этого параметра последовательности является незначительным.

Для подбора оптимальных параметров импульсных РЧ-последовательностей при проведении МРТ-исследований в присутствии различных контрастирующих агентов была разработана программа в среде программирования Delphi. Данная программа наглядно иллюстрирует изменение контраста МР-изображений для различных концентраций контрастных веществ. Рабочее окно программы для проведения МРТ-исследований при отсутствии каких-либо контрастных веществ показано на рис. 4. В окне этой программы отображаются зависимости интенсивности МР-сигнала от различных параметров выбранной последовательности РЧ-импульсов для разных веществ головного мозга. В нем также представлены имитационные МР-изображения, показывающие интенсивность сигнала по шкале „серого“. При изменении пользователем значения параметра последовательности динамически изменяется зависимость интенсивности ЯМР-сигнала от этого параметра и моделируется соответствующее МР-изображение. Это позволяет пользователю выбрать оптимальный контраст МР-изображения, необходимый в конкретном МРТ-исследовании.

Также данная программа позволяет моделировать МР-изображения для МРТ-исследований в присутствии различных контрастных веществ. Активное окно такой программы в случае использования контрастирующих агентов на основе магнитных наночастиц представлено на рис. 5.
Кроме того, разработанная программа дает рекомендации по выбору оптимальной концентрации контрастных веществ в зависимости от цели исследования. Таким образом, пользователь может заранее определить, какая концентрация контрастных веществ должна быть введена для достижения требуемого контраста. Данная функция позволяет уменьшить вред, наносимый организму пациента, за счет минимизации вводимого количества контрастных веществ.

Для лучшего понимания работы импульсных РЧ-последовательностей в МРТ- исследовании в данной программе реализована 3D-анимация, демонстрирующая поведение макроскопической намагниченности и появление эхо-сигнала при использовании конкретной импульсной последовательности. Этот функционал позволяет применять данный программный комплекс не только для практических МРТ-исследований, но и в учебных целях, что расширяет потенциальные сферы практического применения и реализации данной программы.

\section{Выводы}

Таким образом, было исследовано влияние параметров часто используемых в МРТ импульсных РЧ-последовательностей („спиновое эхо“, „инверсия-восстановление“, FLASH) на контраст МР-изображений. В результате была разработана программа для моделирования МР-томограмм и определения оптимальных значений параметров импульсных РЧ-последовательностей для достижения наилучшего контраста МР-изображений. 
Данная программа была апробирована в МРТ-исследованиях с использованием негативных контрастных веществ на основе магнитных наночастиц оксида железа и позитивных контрастных веществ на основе гадолиния. Проанализировано влияние контрастирующих агентов на интенсивность сигнала ЯМР при различных концентрациях этих агентов.

Использование данной программы позволяет сократить время проведения МРТ-исследований, оценить возможность применения различных контрастных веществ для контрастирования МР-изображений. Разработанная программа мониторинга параметров импульсных РЧ-последовательностей при МРТ-исследовании может быть также использована для обучения студентов и медицинского персонала.

\section{Финансирование работы}

Настоящая работа была поддержана Министерством образования и науки Российской Федерации (проект 3.6522.2017).

\section{Конфликт интересов}

Авторы заявляют, что у них нет конфликта интересов.

\section{Список литературы}

[1] Ринкк П.А. Магнитный резонанс в медицине. Основной учебник Европейского форума по магнитному резонансу. М.: ГЭОТАР МЕД, 2003. $247 \mathrm{c}$.

[2] Chizhik V.I., Chernyshev Y.S., Donets A.V., Frolov V.V., Komolkin A.V., Shelyapina M.G. Magnetic Resonance and Its Applications. Springer International Publishing, Switzerland, 2014. $785 \mathrm{p}$.

[3] Богачев Ю.В., Князев М.Н., Марченко Я.Ю. и др. Диагностический магнитный резонанс. СПб: Изд-во СПбГЭТУ „ЛЭТИ“, 2013. $212 \mathrm{c}$.

[4] Богачев Ю.В., Драпкин В.З., Князев М.Н. и др. Магнитнорезонансная томография в слабом магнитном поле. СПб: Изд-во СПбГЭТУ „ЛЭТИ““2012. 260 с.

[5] Анисимов Н.В., Пирогов Ю.А., Губский Л.В., Гладун B.B. Управление контрастом и информационные технологии в магнитно-резонансной томографии, М.: Физический факультет МГУ, 2005. 144 с.

[6] Heywangl S.H., Koebrunner G., Bauer M.W. // Front. Europ. Radiol. 1989. Vol. 6. P. 35-52.

[7] Kuperman $V$. Magnetic resonance imaging physical principles and applications, University of Maryland College Park, Maryland, Academic Press, 2000. 197 p. 